Trauma Berufskrankh $2005 \cdot 7[$ Suppl 1]:S167-S171 DOI 10.1007/s10039-004-0978-2

Online publiziert: 7. Dezember 2004

(c) Springer Medizin Verlag 2004

\section{W. Quirini}

Klinik für Unfall- und Wiederherstellungschirurgie, Allgemeines Krankenhaus Celle

\section{Luxationen des Fußes}

ggf. nochmals bei unklaren Befunden eine Schnittbilddiagnostik, ggf. auch in Form einer Magnetresonanztomographie. Bei der Auswahl des Osteosynthesematerials sollte hierauf Rücksicht genommen werden und in der Regel Titanimplantate verwendet werden.

Die Ruhigstellung erfolgt für insgesamt 6 Wochen. Anschließend werden in Lokal- anästhesie die Drähte und der Fixateur entfernt.

Ergebnisse. Die Spätergebnisse nach subtalarer Luxation sind befriedigend. Gehäuft finden sich jedoch Arthrosen im subtalaren Bereich, die zu Bewegungseinschränkungen und Schmerzen führen. Hansen [3] ist der Ansicht, dass es bei lateralen subtel der Fälle. Hier handelt es sich meistens um eine geschlossene Verletzung. Gehäuft tritt sie als Unfall beim Basketball auf.

Die laterale Luxation betrifft ungefähr ein Drittel der Fälle. Sie findet ihre Ursache gehäuft in Rasanztraumen. Gehäuft finden sich hier auch offene Verletzungen.

Diagnostik. Die Diagnostik umfasst neben der klinischen Untersuchung, insbesondere der Beurteilung der Weichteile, die Anfertigung von Röntgenaufnahmen des Fußes in 2 Ebenen. Bibbo [1] empfiehlt zusätzlich die Durchführung eines Fuß-CT, da bei all seinen Patienten zusätzliche Verletzungen im CT gefunden wurden und dadurch bei $45 \%$ der Patienten ein Wechsel der Behandlung erforderlich wurde.

Therapie. Die Therapie besteht in einer umgehenden Reposition und anschließenden Stabilisation mit K-Drähten und tibiotarsaler Transfixation (• Abb. 1a-d). Die meisten Verletzungen lassen sich geschlossen reponieren. Bei ca. ein Drittel der Fälle treten Repositionshindernisse auf. Dies sind in der Regel Weichteilstrukturen, die eingeschlagen sind oder den Taluskopf umfangen, so z. B. die Tibialis-posterior-Sehne bei der lateralen Luxation oder auch das Retinaculum extensorum bei der medialen Luxation. Hier ist dann ein offenes Vorgehen notwendig.

Nach Reposition und Stabilisierung erfolgt eine nochmalige Röntgenkontrolle,
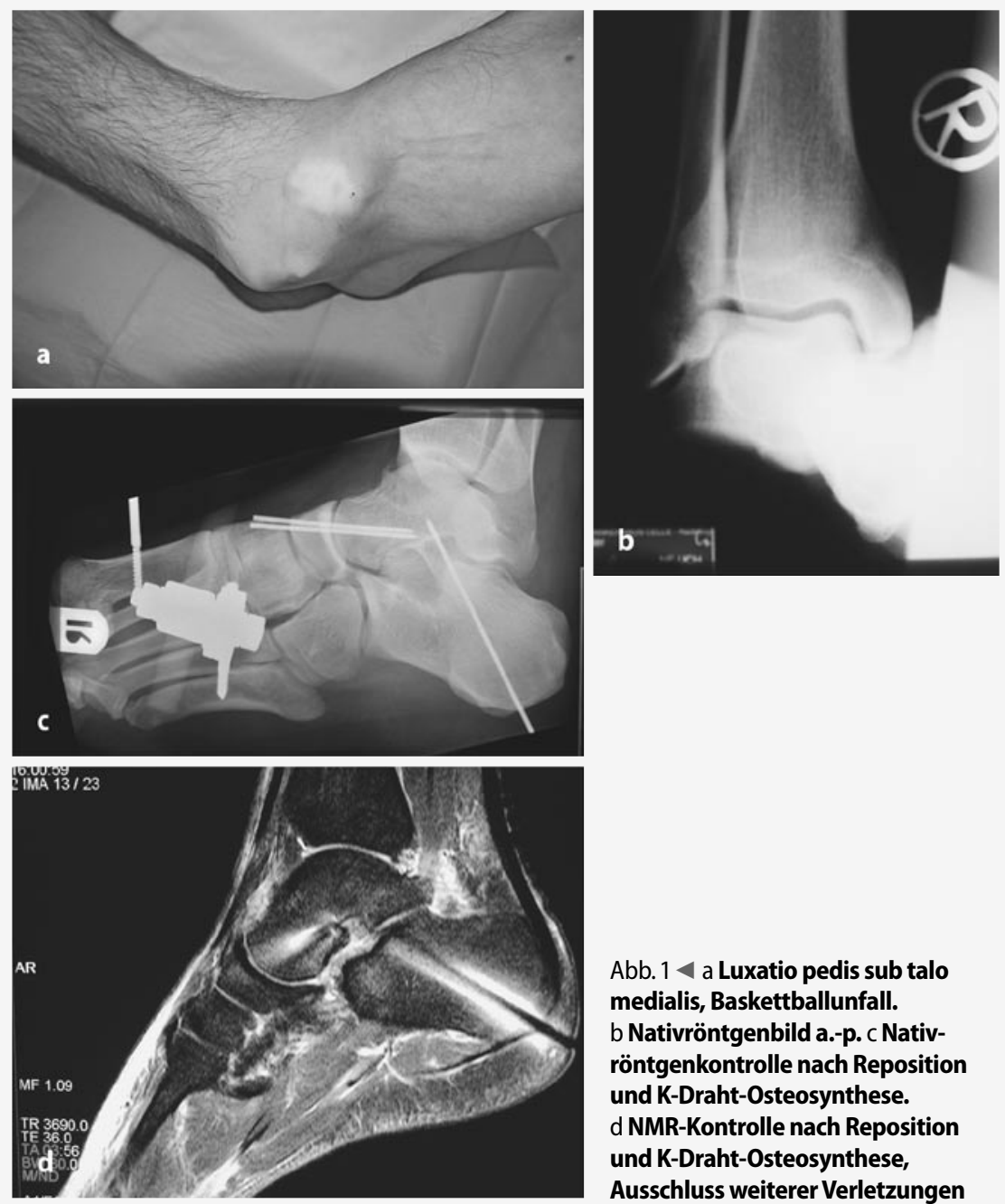

Abb. $1<$ a Luxatio pedis sub talo medialis, Baskettballunfall. b Nativröntgenbild a.-p. c Nativröntgenkontrolle nach Reposition und K-Draht-Osteosynthese. d NMR-Kontrolle nach Reposition und K-Draht-Osteosynthese, Ausschluss weiterer Verletzungen 


\section{Fußverletzungen}

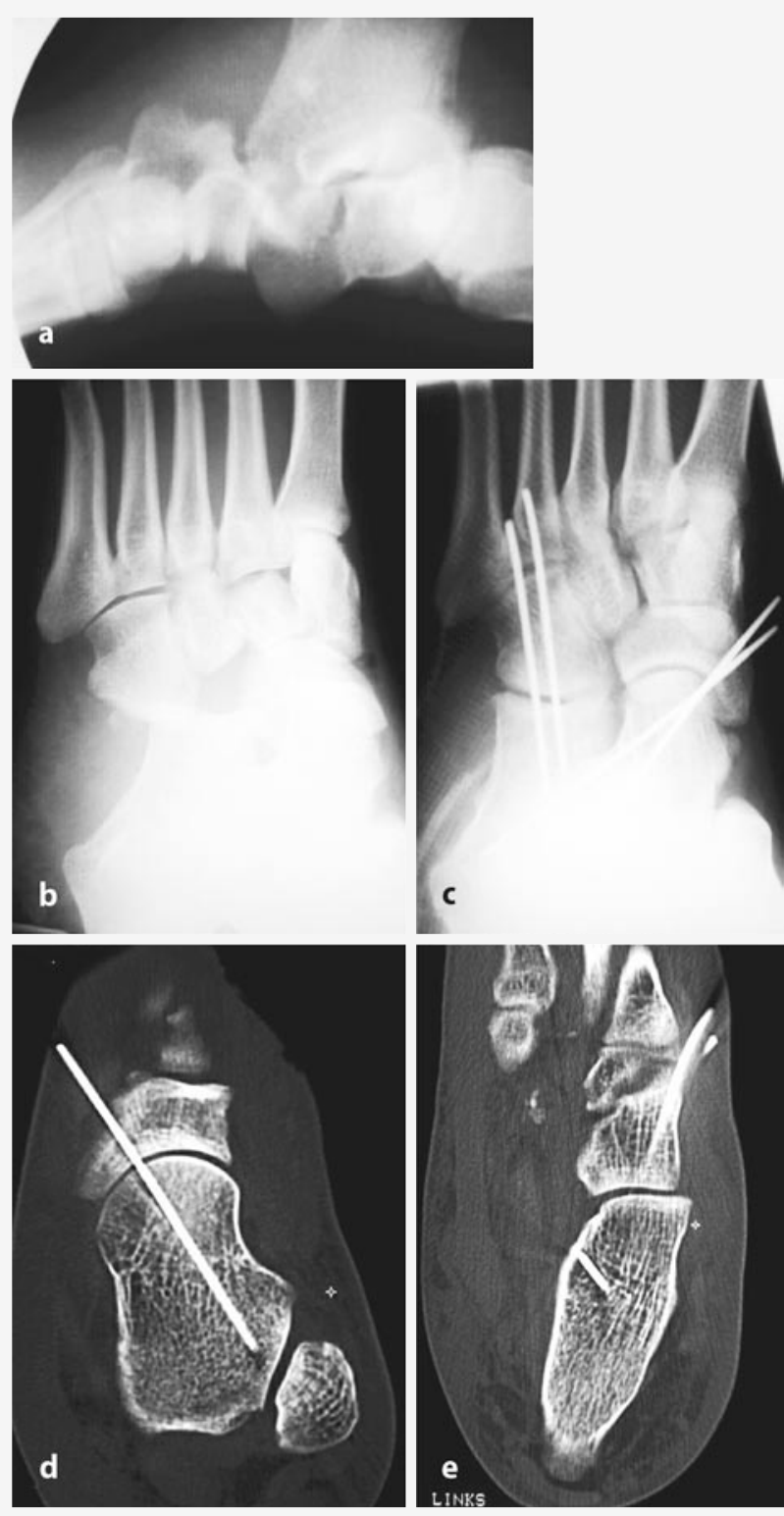

Abb. $2 \Delta$ a Rein ligamentäre Chopart-Luxation, Nativröntgenbild Fuß seitlich. $b$ Nativröntgenbild Fuß dorsoplantar. $c$ Nativröntgen nach geschlossener Reposition und K-Draht-Osteosynthese. d Im CT korrekte Stellung talonavikular. e Im CT korrekte Stellung kalkaneokuboidal

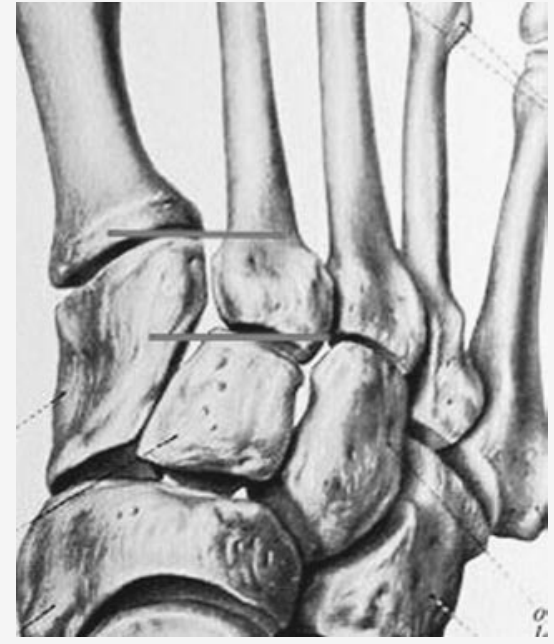

Abb. $3 \Delta$ Tiefe der Lisfranc-Gabel
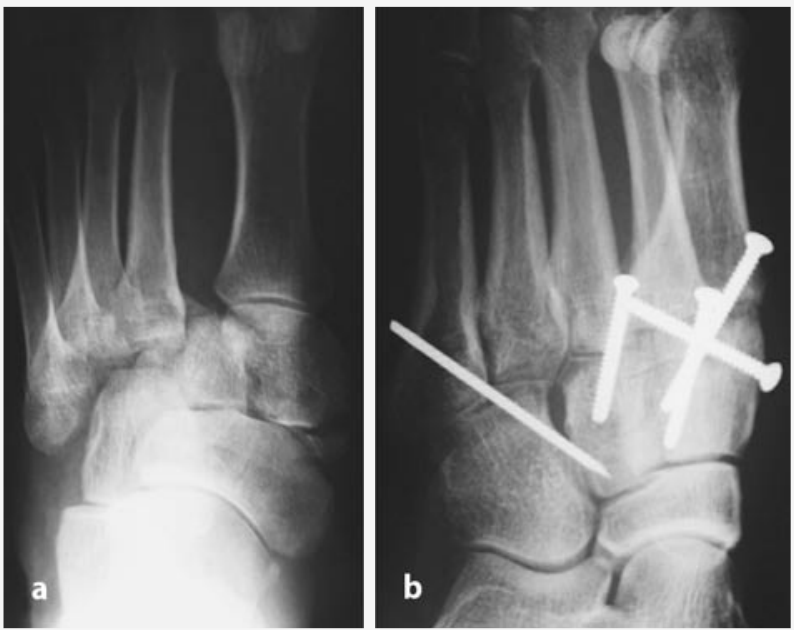

Abb. $4 \Delta$ a Lisfranc-Luxation, dorsoplantare Aufnahme. b Nach offener Schraubenosteosynthese der Strahlen 1-3 und K-Draht-Fixation der lateralen Strahlen talaren Luxationen generell ein schlechteres Ergebnis gibt, da gehäuft Verletzungen auch des N. tibialis oder der Tibialis-posterior-Sehne vorhanden seien, die das Spätergebnis beeinträchtigen. Bibbo [2] und auch Perugia [11] kommen jedoch bei ihren Arbeiten zu dem Ergebnis, dass sowohl für laterale als auch für mediale Luxationen ein gleiches Outcome besteht.

\section{Chopart-Luxationen}

Dislokationen in der Chopart-Gelenkreihe sind aufgrund der starken kapsoliga- mentären Führung der Gelenkreihe immer Ausdruck einer extremen Gewalteinwirkung auf den Fuß. Dem Vorschlag von Zwipp [14] folgend, unterscheidet man 6 verschiedene Formen der Chopart-Luxation, wobei die rein transligamentäre Verletzung extrem selten ist. Die übrigen Formen entstehen durch knöcherne Mitbeteiligung der angrenzenden Fußwurzelknochen in das Dislokationsgeschehen.

Diagnostik. Die Diagnostik umfasst neben der klinischen Untersuchung, insbesondere des Erkennens der Weichteilsitua- tion, die radiologische Diagnostik. Hierbei sind 3 Röntgenaufnahmen erforderlich zum einen die Aufnahme des Fußes dorsoplantar mit um $30^{\circ} \mathrm{kraniokaudal} \mathrm{gekipp-}$ ter Röntgenröhre, die exakt seitliche Position des Fußes und die $45^{\circ}$-Schrägaufnahme des Fußes. Im eigenen Vorgehen wird bei allen Chopart-Luxationen zusätzlich ein Fuß-CT durchgeführt.

Behandlung. Die Ziele der Behandlung einer Chopart-Luxation sind zum einen das Wiederherstellen der Gelenkflächen, der Schutz des Bandapparates und der Weich- 
teile, aber insbesondere die Wiederherstellung der Länge der medialen und der lateralen Säule des Fußes. Der letzte Punkt ist von entscheidender Bedeutung. So konnte Mittlmeier [8] in seiner experimentellen Arbeit zeigen, dass die spätere Funktion des Fußes, insbesondere die Gangfunktion, nicht durch den radiologischen Grad der Arthrose beeinflusst wird, sondern durch einen Längenverlust der medialen oder lateralen Säule oder ein Verschieben der mechanischen Achse in horizontaler oder vertikaler Ebene.

Die Behandlung hat sich an diesen Grundsätzen auszurichten. Konservativ behandelt werden können daher nur undislozierte Frakturen im Bereich der Chopart-Linie oder Subluxationen kalkaneokuboidal und talonavikular und reponierte komplette Luxationen, wenn sie reponibel sind und sicher retiniert werden können. Alle anderen Verletzungen müssen nach den oben genannten Grundsätzen operativ versorgt werden.

Alle Luxationen, die zwar geschlossen zu reponieren sind, aber anschließend nicht stabil sind, sollten mit einer perkutanen Kirschner-Draht-Osteosynthese versorgt werden. Die Drähte sollten bei gleichzeitiger Gipsruhigstellung des Fußes (• Abb. 2ae) für 6 Wochen belassen bleiben.

Alle Luxationsformen, die geschlossen nicht reponiert werden können oder Gelenkstufen bzw. eine Verkürzung der medialen oder lateralen Säule zeigen, sollten offen operativ versorgt werden.

Die Nachbehandlung besteht in der Regel in einer Ruhigstellung im Gipsverband für 6 Wochen. Anschließend Entfernung der Kirschner-Drähte in Lokalanästhesie und die Vollbelastung in der Regel nach 8-12 Wochen.

Ergebnisse. Die Spätergebnisse sind trotz der differenzierten Behandlung eher mäßig. Kotter [5] fand bei der Nachuntersuchung seiner Patienten, dass in 60\% der Fälle ein mäßiges und schlechtes Ergebnis zu finden war, dass nur ein Viertel der Patienten das Arbeitsniveau der Zeit vor dem Unfall erreichten und keiner der Patienten das sportliche Niveau aus der Zeit vor dem Unfall.

\section{Lisfranc-Luxationen}

Luxationen der tarsometatarsalen Gelenkreihe beruhen in der Regel auf einer indi-
Trauma Berufskrankh 2005 · 7[Suppl 1]:S167-S171

DOI 10.1007/s10039-004-0978-2

c) Springer Medizin Verlag 2004

W. Quirini

\section{Luxationen des Fußes}

\section{Zusammenfassung}

Luxationen der großen Gelenkreihen des

Fußes sind seltene Verletzungen, stellen jedoch in der Regel eine dringliche Operationsindikation dar. Schnittbildverfahren sollten bei der Diagnostik und zur Kontrolle des postoperativen Ergebnisses großzügig eingesetzt werden. Bei der Implantatwahl sollte an die spätere Schnittbilddiagnostik gedacht werden. Es empfiehlt sich daher der vermehrte Einsatz von Titanimplantaten. Grundvoraussetzung für ein gutes Outcome ist die exakte anatomische Reposition der Gelenkflächen und die korrekte Wiederherstellung der Längen und Achsen

\section{Dislocations of the foot}

\section{Abstract}

Dislocations of the major joints of the foot are rare injuries, but they usually require urgent surgery. Imaging methods should be used generously in diagnostics and for postoperative follow-up, and should be taken in consideration when chosing the implant. Therefore the increased use of titanium implants is recommended. The basic requirements for a successful outcome are the exact anatomical reconstruction of the joint surfaces, the foot length and axes. Treatment of Lisfranc's dislocation tends to have des Fußes. Bei der Versorgung von Verletzungen der Lisfranc-Reihe hat die Schraubenosteosynthese ein tendenziell besseres Spätergebnis als die perkutane KirschnerDraht-Osteosynthese. Das Kompartmentsyndrom des Fußes stellt eine häufige Komplikation der Luxationen der großen Gelenkreihe des Fußes dar und muss rechtzeitig erkannt werden.

\section{Schlüsselwörter}

Große Gelenkreihe des Fußes .

Subtalare Luxation - Lisfranc-Luxation .

Chopart-Luxation · Kompartmentsyndrom

better long-term results when using screw fixation rather than K-wire osteosynthesis. Common complication of injuries of the major foot joints is the compartment syndrome, which must be timely recognized.

\section{Keywords}

Major joint series of the foot .

Subtalar dislocation - Lisfranc's dislocation . Chopart's dislocation .

Compartment syndrome 
rekten Gewalteinwirkung, einen Sturz auf den plantar-flektierten Fuß oder ein Dezelerationstrauma im Fußraum des PKW. Die Einteilung erfolgt nach der Einteilung von Quenú [15] sowie den Vorschlägen von Hardcastle [4] in einen homolateralen Typ, in einen divergierenden Typ und in einen isolierten Typ.

Peicha [10] konnte in einer vergleichenden Arbeit feststellen, dass auch die individuelle Gelenkanatomie als Risikofaktor für das Auftreten einer Lisfranc-Luxation anzusehen ist. In einer vergleichenden Studie konnte er zeigen, dass das Risiko einer Lisfranc-Luxation steigt mit der Abnahme der Tiefe der sog. Lisfranc-Gabel zwischen der Basis des 1. und 2. Mittelfußknochens (- Abb. 3).

Diagnostik. Die Diagnostik einer Lisfranc-Luxation umfasst zum einen den klinischen Befund, hier ist insbesondere auf das Auftreten eine Kompartmentsyndroms zu achten. Die konventionellen Röntgenaufnahmen sollten in 3 Ebenen erfolgen: Zum einen die exakt seitliche Ebene, zum anderen die dorsoplantare Aufnahme mit um $20^{\circ}$ kaudal-kranial gekippter Röhre sowie die $45^{\circ}$-Schrägaufnahme des Fußes. Darüber hinaus ist jedoch in eigentlich allen Fällen die Anfertigung eines Fuß-CT zu fordern. Peicha [9] konnte in seiner Arbeit ganz klar die Überlegenheit der Schnittbilddiagnostik gegenüber dem konventionellen Röntgenbild bei der Evaluation von Verletzungen der LisfrancReihe zeigen.

Darüber hinaus konnte Lu [7] in seiner experimentellen Arbeit nachweisen, dass Dislokationen von $1 \mathrm{~mm}$ im Bereich der Lisfranc-Reihe im Nativröntgen zu 100\% nicht zu erkennen waren, Dislokationen von $2 \mathrm{~mm}$ im Röntgen $\mathrm{zu}$ 70\% nicht erkennbar waren. Dies belegt eindeutig die Überlegenheit des CT im Vergleich zu den konventionellen Röntgenaufnahmen.

Behandlung. Ziel der Behandlung der Lisfranc-Luxation muss die anatomische Rekonstruktion der Gelenkflächen sein und die Wiederherstellung der Stabilität. Daher ist eine konservative Therapie nur in Ausnahmefällen indiziert. Bei der operativen Behandlung stehen sich offene und geschlossene Verfahren gegenüber. Bei der geschlossenen Reposition und Stabili- sierung perkutan mit Kirschner-Drähten handelt es sich um eine sehr weichteilschonende und schnelle Methode. Die Exaktheit der Reposition ist jedoch nur radiologisch zu beurteilen, und auch ein möglicherweise drohendes Kompartmentsyndrom lässt sich hiermit nicht behandeln. Für die offene Reposition und Schraubenosteosynthese spricht die Exaktheit der Versorgung sowie die höhere Primär- und Sekundärstabilität. Des Weiteren ergibt sich durch den operativen Zugang eine deutliche Druckentlastung der Weichteile.

Ergebnisse. Für das spätere Outcome ist die Exaktheit der Reposition der entscheidende Faktor. Dies belegen Übersichtsarbeiten von Richter [12] und Kuo [6]. Richter [12] fand bei der Auswertung seiner Fälle ein tendenziell besseres Outcome bei offener Reposition und Schraubenosteosynthese.

Bei einem Teil der Patienten resultiert trotz anatomischer Reposition der Gelenkflächen doch ein schlechtes Spätergebnis. Kuo [6] ist der Auffassung, dass die schlechteren Spätergebnisse bei rein ligamentärer Verletzung zu erwarten sind. Teng [13] kann bei den von ihm untersuchten Fällen die Ursache hierfür nicht erfassen.

Die Nachbehandlung besteht bei geschlossenem Verfahren und KirschnerDraht-Osteosynthese in einer 6-wöchigen Gipsruhigstellung, Entfernen der K-Drähte nach ca. 8 Wochen und schrittweiser Belastungsaufbau mit Erreichen der Vollbelastung nach ca. 10-12 Wochen. Die mittels stabiler Schraubenosteosynthese versorgten Fälle können bei guter Compliance auch gipsfrei nachbehandelt werden. Das Erreichen der Vollbelastung ist jedoch auch in diesen Fällen nicht früher möglich (• Abb. 4a, b)

\section{Kompartmentsyndrom}

Das Kompartmentsyndrom ist eine häufige Komplikation von Luxationen im Bereich des Fußes. Die Diagnose wird klinisch aufgrund der massiven Schwellung mit Sensibilitäts- und Funktionsstörungen im Bereich der Zehen gestellt. Die Kompartmentdruckmessung ist sicherlich ein Hilfsmittel. Werte über $25 \mathrm{mmHg}$ sind als pathologisch anzusehen. Die Behandlung erfolgt durch Spaltung der Komparti- mente des Fußes über eine dorsale gerade Inzision mit zusätzlicher Durchtrennung der Retinakula. Gelegentlich kann auch die 2-Schnitt-Technik mit Inzision über dem Zwischenraum zwischen dem 1. und 2. Strahl und über dem Zwischenraum zwischen 4. und 5. Strahl erfolgen.

\section{Fazit}

Zwar sind Luxationen der großen Gelenkreihen des Fußes Verletzungen, die selten vorkommen, doch stellen sie in der Regel eine dringliche Operationsindikation dar. Der begleitende Weichteilschaden muss exakt erfasst werden, um insbesondere ein Kompartmentsyndrom zu erkennen.

\section{Korrespondierender Autor Dr. W. Quirini}

Klinik für Unfall- und Wiederherstellungschirurgie, Allgemeines Krankenhaus, Siemensplatz 4, 29223 Celle E-Mail:Wolfgang.Quirini@akh-celle.de

Interessenkonflikt: Der korrespondierende Autor versichert, dass keine Verbindungen mit einer Firma, deren Produkt in dem Artikel genannt ist, oder einer Firma, die ein Konkurrenzprodukt vertreibt, bestehen.

\section{Literatur}

1. Bibbo C, Lin SS, Abidi N et al. (2001) Missed and associated injuries after subtalar dislocation: the role of CT. Foot Ankle Int 22: 321-328

2. Bibbo C, Anderson RB, Davis WH (2003) Injury characteristics and the clinical outcome of subtalar dislocations: a clinical and radiographic analysis of 25 cases. Foot Ankle Int 24: 158-163

3. Hansen ST (2000) Functional and reconstructive surgery of the foot and ankle. Lippincott Williams \&Wilkins, Philadelphia

4. Hardcastle PH, Reschauer R, Kutscha-Lissberg E, Schoffmann W (1982) Injuries to the tarsometatarsal joint: Incidence, classification and treatment. J Bone Joint Surg 64-B: 349-356

5. Kotter A, Wieberneit J, Braun W, Rüter A (1997) Die Chopart-Luxation - eine häufig unterschätzte Verletzung und ihre Folgen. Unfallchirurg 100: 742749

6. Kuo RS, Tejurani NC, Digiovanni CW et al. (2000) Outcome after open reduction and internal fixation of Lisfranc joint injuries. J Bone Joint Surg 82-A: 1609-1618

7. Lu J, Ebraheim NA, Skie M et al. (1997) Radiographic and computed tomographic evaluation of Lisfranc dislocation - a cadaver study. Foot Ankle Int 18: $351-355$ 
8. Mittlmeier T, Krowiorsch R, Brosinger S, Hudde M (1997) Gait function after fracture-dislocation of the midtarsal and/or tarsometatarsal joints. Clin Biochem 12: 516-517

9. Peicha G, Preidler UW, Lajtai G et al. (2001) Diagnostischer Wert von konventionellen Röntgenaufnahmen, Computertomographie und Kernspintomographie bei akuten Verletzungen des Fußes. Unfallchirurg 104: 1134-1139

10. Peicha G, Labovits J, Seibert FJ et al. (2002) The anatomy of the joint as a risk factor for Lisfranc dislocation and frature-dislocation. An anatomical and radiological case control study. J Bone Joint Surg 84B: $981-985$

11. Perugia D, Basile A, Massoni C et al. (2002) Conservative treatment of subtalar dislocation. Int Orthop 26: 56-60

12. Richter M, Wippermann D, Krettek C et al. (2001) Fractures and fracture-dislocations of the midfoot: occurence, causes and long-term results. Foot Ankle Int 22: 392-398

13. Teng AL, Pinzur MS, Lomasney L et al. (2002) Functional outcome following anatomic restauration of tarsal- metatarsal fracture dislocation. Foot Ankle Int 23: 922-926

14. Zwipp H (1994) Chirurgie des Fußes. Springer, Wien New York

15. Quenú E, Küss $G$ (1909) Études sur les luxationes du metatarse. Rev Chir 39: 281, 720, 1093 